\title{
P38a Deficiency in Macrophages Ameliorates Murine Experimental Colitis by Regulating Inflammation and Immune Process
}

\section{Wei Chen}

Shanghai Jiao Tong University Affiliated Sixth People Hospital

\section{Rui Liang}

Shanghai Jiao Tong University Affiliated Sixth People Hospital

\section{Youcai Yi}

Shanghai Jiao Tong University Affiliated Sixth People Hospital

\section{Xiaoyu Chen}

Shanghai Jiao Tong University Affiliated Sixth People Hospital

\section{Huining Fan}

Shanghai Jiao Tong University Affiliated Sixth People Hospital

Jin-Shui Zhu ( $\nabla$ zhujs1803@163.com )

Shanghai Jiao Tong University Affiliated Sixth People Hospital

\section{Jing Zhang}

Shanghai Jiao Tong University Affiliated Sixth People Hospital

\section{Research Article}

Keywords: p38 MAPK, colitis, macrophages, immune process, inflammation response

Posted Date: July 19th, 2021

DOI: https://doi.org/10.21203/rs.3.rs-563958/v2

License: (c) (i) This work is licensed under a Creative Commons Attribution 4.0 International License.

Read Full License

Version of Record: A version of this preprint was published at Pathology - Research and Practice on April 1st, 2022. See the published version at https://doi.org/10.1016/j.prp.2022.153881. 


\section{Abstract}

Introduction: P38a is a mitogen-activated protein kinase (MAPK) that mediates inflammatory responses. P38a alterations have been associated with the inflammation-related diseases. However, the role of macrophages-derived p38a in dextran sulfate sodium (DSS)-induced murine experimental colitis remains unclear.

Objectives: We characterized the role of macrophages-derived p38a in DSS-induced colitis.

Methods: The expression of macrophage-derived p38a in human colitis and normal tissues was measured by immunohistochemistry $(\mathrm{IHC})$ and fluorescence in situ hybridization (FISH) analysis. Macrophage-specific p38a knockout (p38a ${ }^{\Delta M \varphi}$ ) and wild type (WT) mice administrated by $3 \%$ DSS were used to establish experimental colitis. The alterations in inflammatory cytokines, intestinal epithelial barrier, cell proliferation and cell apoptosis between $\mathrm{p} 38 \mathrm{a}^{\Delta \mathrm{M} \varphi}$ and WT groups were determined by IHC, immunofluorescence (IF), TdT-mediated dUTP Nick-End Labeling (TUNEL) and Western blot analyses. The enriched pathways between $\mathrm{p} 38 \mathrm{a}^{\Delta \mathrm{M} \varphi}$ and WT groups were identified by RNA-seq and KEGG analysis. SB203580 and BIRB796 as the p38 MAPK inhibitors were used to treat DSS-induced colitis.

Results: p38a was co-localized with CD68 in the cytoplasm and their co-expression indicated an increased level in colitis tissues as compared with the normal tissues. P38a deficiency in macrophages was sufficient to suppress the exacerbated clinical symptoms and inflammation responses in experimental colitis, followed by reducing cytokine release, increasing MUC-2 and Claudin-2 secretion and promoting colonic mucosa repair. Further investigations validated that the immune process-related factors such as Lgals9, Rtp4, Ddx60, Nlrp1b, Hsh2d, Oas2 and Oas3 were upregulated in colon tissues from $p 38 a^{\Delta M \varphi}$ group as compared with the WT group. Inhibition of p38 MAPK attenuated DSS-induced colitis.

Conclusion: Our findings demonstrated that p38a deficiency in macrophages ameliorated murine experimental colitis by regulating inflammation and immune process.

\section{Introduction}

Inflammatory bowel disease (IBD), including ulcerative colitis (UC) and Crohn's disease (CD), is characterized by chronic and recurrent inflammatory and immune infiltration in gastrointestinal tract (Xavier and Podolsky 2007). Increasing evidence unveils that the pathogenesis of IBD is associated with multiple factors such as genetic polymorphisms, environmental factors, and immunological disorders (Cha, et al. 2017; Shi, et al. 2018). A better understanding of the molecular pathogenesis of IBD is critical to develop promising therapeutic strategies for this disease.

The core of the inflammatory signaling is mediated by reversible phosphorylation by protein kinases, of which mitogen-activated protein kinase (MAPK) signaling acts in linking inflammatory stimuli to cellular responses (Kim, et al. 2008). The p38 MAPKs are a family of serine/threonine protein kinases that consist 
of four isoforms p38a, p38 3 , p38y and p38ס (Kumar, et al. 2003a), of which p38a is the most commonly expressed in human and mouse tissues (Jiang, et al. 1997) and controls inflammatory response and cancer cell proliferation (Hui, et al. 2007; Kumar, et al. 2003a). Hepatocyte-derived p38a is a pivotal negative regulator in maintaining hepatic gluconeogenesis (Jing, et al. 2015). P38a appears to have dual roles in certain cancers. It suppresses colitis-associated tumor initiation, but contributes to the proliferation and survival of colon cancer (Gupta, et al. 2014). P38a deletion in myeloid cells improves experimental colitis, but its deficiency in intestinal epithelial cells (IECs) increases the susceptibility to colitis (Otsuka, et al. 2010).

Emerging evidence indicates that macrophage-specific p38a accelerates acute liver injury (Liu, et al. 2017) and steatohepatitis progression through inducing pro-inflammatory cytokine secretion and M1 polarization (Zhang, et al. 2019). However, the effects of macrophage-derived p38a on IBD pathogenesis remain undocumented. In the present study, p38a was co-localized with CD68 in the cytoplasm and their co-expression displayed an increased level in colitis tissues. We then used macrophage-specific p38a knockout mice (p38a $\mathrm{a}^{\Delta \mathrm{M} \varphi}$ ) and WT mice to construct DSS-induced experimental colitis and found that p38a deficiency in macrophages ameliorated murine experimental colitis by inhibiting cytokine release, promoting colonic mucosa repair and regulating immune process.

\section{Materials And Methods}

\section{Tissue sample}

10 cases of colitis and 5 normal colon tissues were purchased from Xi'an Best Biotechnology Co., Ltd. (Xi'an, China).

\section{Animals and treatments}

C57BL/ 6 male p38 $a^{\Delta M \varphi}$ mice and wild type (WT) littermates (6-8 weeks of age) were provided by Medical College of Xiamen University and housed in the SPF standard laboratory conditions.

As previously described (Weigmann, et al. 2017), p38a $a^{\Delta M \varphi}$ mice $(n=5)$ and WT mice $(n=6)$ were administrated by the addition of $3 \%$ DSS (36-50 kDa, MP Biomedicals, USA) in the drinking water for 7 consecutive days. In another cohort, male C57BL/6 mice (6-8 weeks old) were intraperitoneally injected with p38 MAPK inhibitors or vehicle (DMSO) for 7 consecutive days. According to previous literature (Zhang, et al. 2019), the injection doses of p38 MAPK inhibitors SB203580 and BIRB796 were 50 $\mathrm{mg} / \mathrm{kg} /$ day and $15 \mathrm{mg} / \mathrm{kg} /$ day, respectively. Mouse body weight, colon length, clinical phenotype and pathological scoring were assessed.

\section{Evaluation of DSS-induced colitis}

The mice were observed every morning. Weight loss, fecal consistency and intestinal bleeding were recorded in accordance with the previous report (Weigmann, et al. 2017). The DAl value was evaluated 
based on the above records.

\section{Histopathological assessment}

The colon from the anus to the cecum was obtained and measured. The lower colon tissues in each group were fixed in paraformaldehyde solution (4\%) for 48 hours, and then embedded in paraffin. Histopathological assessment of the inflammatory responses in colon tissues in each group was determined by hematoxylin and eosin (H\&E) staining in accordance with the previous report (Chen, et al. 2011).

\section{Immunohistochemistry and immunofluorescence}

Immunohistochemistry (IHC) and immunofluorescence (IF) analyses were performed to evaluate the protein levels of claudin-2 (AF0128, Affinity Biosciences, USA), MUC-2 (DF8390, Affinity Biosciences, USA), OAS2 (DF12680, Affinity Biosciences, USA), OAS3 (ER1914-27, HuaBio, China), P53 (AF0879, Affinity Biosciences, USA), ki-67 (ab16667, Abcam, United Kingdom), c-myc (AF0358, Affinity Biosciences, USA), TNF-a (17590-1-ap, Proteintech, China), IL-1ß (ab33591, Lianke Biotechnology, China), IL-6 (218651-AP, Proteintech, China), and p-p38 (AF4001, Affinity Biosciences, USA), in paraffin-embedded colonic sections in each group. The detailed description of IHC and IF procedure is performed according to the previous reports (Wang, et al. 2017)' (Chang, et al. 2019). The sum of the area\% was analyzed by ImageJ software.

\section{RNA sequencing and bioinformatic analysis}

Briefly, total RNA was extracted from the colon tissues of the WT and p38a ${ }^{\Delta M \varphi}$ groups. The quantitative and quality control of total RNA were detected by NanoDrop ND-1000. 1-2 $\mu$ g RNA was extracted from each sample to generate the RNA library, which were sequenced on an Illumina Hiseq 4000 platform after cluster generation. Data were analyzed as follows: sequencing quality control, sequencing data comparison, quantitative analysis of differentially-expressed genes, and GO and KEGG enrichment analysis.

\section{Quantitative Real-time PCR (qRT-PCR)}

Trizol reagent (Invitrogen, USA) was used to isolate total RNA from colon tissues Complementary DNA (cDNA) is obtained by RNA reverse transcription. The reaction procedure is based on our previous reports (Chen, et al. 2019). The $2^{-\Delta \Delta C t}$ method was used to calculate the gene expression level. GAPDH was used as the internal control. All reactions were performed more than twice. All primers were listed in Table S1.

\section{Western blot analysis}

The protein levels of Claudin-2, OAS2 and OAS3 in colon tissues in each group were detected by Western blotting. In briefly, total proteins from colon tissues were obtained with RIPA lysis buffer (Beyotime, Shanghai, China) and protease inhibitor (Servicebio, China). After the determination of protein 
concentration, the protein was denatured by boiling water for 15 minutes. The primary antibodies claudin2 (AF0128, Affinity Biosciences, USA), OAS2 (DF12680, Affinity Biosciences, USA), OAS3 (ER1914-27, HuaBio, China), and anti- $\beta$-actin (AF7018, Affinity Biosciences, USA) were thinned and incubated overnight at $4{ }^{\circ} \mathrm{C}$. We added HRP-labeled goat anti-rabbit secondary antibodies in a 1:3000 dilution, and incubated them at room temperature for 30 minutes.

Wash the membranes three times with PBST. The immunoreactive bands were detected with ECL Luminescent Solution (Servicebio, China).

\section{TdT-mediated dUTP Nick-End Labeling (TUNEL) Assay}

Apoptosis of IECs was detected by TUNEL kit (Servicebio, China). In short, colon tissue sections were cultured in TUNEL reaction mixture. Then, it was stained with 4', 6-diamino-2-phenylindole (DAPI). After that, all samples were observed under the fluorescence inverted microscope. Each sample was observed in three random fields.

\section{Fluorescence in situ hybridization (FISH) analysis}

Oligonucleotide modified probe sequences for P38a (probe1: 5'-Dig-CGGGCACCTCCCAGATTGTCTTGTT 3', probe2: 5'-Dig-GCCGAGCCAGTCCAAAATCCAGAAT 3', probe3: 5'-Dig-TCCAATACAAGCATCTTCTCCAGC 3') and CD68 (probe1: 5'-Bio-GGGGAATGAGAGAAGCAGGTGGGG 3', probe2: 5'-BioGCAATAAGCACCAGGGCGAGGAGGC 3', Probe3: 5'-Bio-GCCGAGAATGTCCACTGTGCTGCGT 3') were applied for FISH. The detailed operation steps were performed as described above (Wang, et al. 2018). The images were analyzed by pathological image quantitative analysis-HALO software (Indica Labs, USA).

\section{Statistical analysis}

Statistical analyses were performed with SPSS 20.0 and GraphPad Prism 8.0.1. All data were displayed as the mean \pm SEM. The differences between groups were analyzed by One-way ANOVA. $P<0.05$ means statistical difference.

\section{Results}

\section{The expression of macrophage-derived p38a is increased in human colitis tissues}

The protein levels of phosphorylated p38a (p-p38a) in colitis and normal tissues were detected by IHC analysis, which indicated that, $\mathrm{p}$-p38a presented no differential expression between colitis and normal tissues ( $P=0.1363$, Fig. $1 \mathrm{~A}, \mathrm{~B})$. Further investigations showed that p38a was co-localized with CD68 in the cytoplasm of colitis tissue cells (Fig. 1C) and they harbored a positive correlation in colitis tissues $\left(R^{2}=0.5015\right.$, Fig. 1D). FISH analysis also confirmed that p38a or CD 68 possessed no differential expression between colitis and normal tissues, but the co-expression level of p38a and CD68 were significantly higher than those in normal tissues ( $P=0.0312 \varangle$ Fig. 1E). 
To understand how macrophage-derived p38a leads to the severe colitis, we used $p 38 a^{\Delta M \varphi}$ and WT mice to establish DSS-induced experimental colitis. The body weight changes, stool consistency, intestinal bleeding and colon length were observed. The results showed that, with or without DSS intervention, the body weight was increased, but DAI was decreased in $\mathrm{p} 38 \mathrm{a}^{\Delta \mathrm{M} \varphi}$ group as compared with the WT group (Fig. 2A, B). Likewise, after exposure to DSS treatment, the colon length was raised in $p 38 a^{\Delta M \varphi}$ group as compared with the WT group (Fig. 2C, D). Histological examination of colon sections showed that p38 $\mathrm{a}^{\Delta \mathrm{M} \varphi}$ mice administrated by DSS displayed a lower histopathological scoring in colon tissues accompanied by less neutrophil infiltration, submucosal edema, and loss of crypt and goblet cell as compared with WT mice (Fig. 2E, F).

\section{Macrophage-specific knockout of p38a inhibits pro-inflammatory cytokine secretion in experimental colitis}

To clarify the effects of macrophage-derived p38a on proinflammatory cytokine production in experimental colitis, we investigated the levels of TNF- $a$, IL-1 $\beta$ and IL-6 in p38 $a^{\Delta M \varphi}$ and WT groups. Previous studies have indicated that the secretion of cytokines TNF- $\alpha$, IL-1 $1 \beta$ and IL- 6 was associated with the pathogenesis of IBD (Hanauer 2006; Souza and Fiocchi 2016). IHC analysis indicated that the expression levels of TNF-a, IL-1 $\beta$ and IL- 6 in experimental colitis in $p 38 a^{\Delta M \varphi}$ group were significantly lower than those in the WT group (Fig. 3A-C).

\section{Macrophage-specific knockout of p38a promotes intestinal barrier repair in experimental colitis}

Intestinal mucosal integrity can be altered during active colitis, leading to mucosal metabolism disorder and aggravated colon inflammation (Michielan and D'Incà 2015). To understand how p38a in macrophages affects intestinal barrier function, we tested the tissue levels of mucosal barrier-related markers such as occludin, ZO-1, claudin-1, claudin-2, muc-1 and muc-2 in experimental colitis in p38 $\mathrm{a}^{\triangle \mathrm{M} \varphi}$ and WT groups. qRT-PCR and IHC analysis displayed that occludin, ZO-1, claudin-1 and muc-1 harbored no differential expression (Supplementary Fig. S1), but claudin-2 and muc-2 were upregulated in p38a ${ }^{\Delta M \varphi}$ group as compared with the WT group (Fig. 4A, B). The similar results were further validated by Western blot and IF analyses in $\mathrm{p} 38 \mathrm{a}^{\Delta \mathrm{M} \varphi}$ and WT groups. (Fig. 4C, D).

\section{Macrophage-specific knockout of p38a regulates the proliferation and apoptosis of IECs in experimental colitis}

Accumulating data show that p38a is related to cell proliferation in IBD (Otsuka, et al. 2010). To determine the effect of p38a in macrophages on the proliferation of IECs, ki-67 proliferation index and cell apoptosis in experimental colitis were measured by IHC and TUNEL, which indicated that ki-67 proliferation index of IECs was increased (Fig. 5A), whereas cell apoptosis was decreased in p38a $a^{\Delta M \varphi}$ group as compared with the WT group (Fig. 5B). 
To further dissect the underlying mechanisms of macrophage-derived p38a in experimental colitis, we performed a RNA-seq analysis in experimental colitis between $p 38 a^{\Delta M \varphi}$ and WT groups. Heatmaps and volcano analysis revealed that 53 differentially-expressed genes were identified between these two groups (Fig. 6A, B). The results of $\mathrm{GO}$ analysis revealed that these differential genes were enriched in multiple biological processes, of which immune system process ranked the second place and aroused our attention (Fig. 6C, D). The related genes were shown in Figure 6E. We then validated the expression of these immune system process-related genes between $p 38 a^{\Delta M \varphi}$ and WT groups by qRT-PCR analysis. The results indicated that the mRNA levels of Lgals9, Rtp4, Ddx60, Nlrp1b, Hsh2d, Oas2 and Oas3 were upregulated in $\mathrm{p} 38 \mathrm{a}^{\Delta \mathrm{M} \varphi}$ group compared with the WT group (Fig. 6F). The protein levels of Oas2 and Oas3 were further validated by IHC analysis (Fig. 6G).

\section{The p38 MAPK inhibitor improves DSS-induced experimental colitis}

To assess the effects of targeting p38 MAPK on DSS-induced colitis, we used p38 MAPK inhibitors SB203580 and BIRB796 to treat DSS-induced colitis. It was found that the mice in SB203580 group gained an increasement of weight and decline of DAI scoring compared with the DSS group (Fig. 7A, B). Meanwhile, the colon length was increased in SB203580 group as compared with the DSS group (Fig. 7C, D). Histopathological examination demonstrated that SB203580 could inhibit a higher colon pathological scoring in experimental colitis, accompanied by less neutrophil infiltration, loss of crypt and goblet cell, and submucosal edema as compared to with DSS group (Fig. 7E, F).

\section{Discussion}

It has been reported that macrophage-derived p38a promotes liver injury and steatohepatitis progression. Herein, we estimated the role of macrophage-derived p38a in DSS-induced experimental colitis. We found that the expression of macrophage-derived p38a was elevated in colitis tissues, and macrophage-specific knockout of p38a improved the clinical symptoms, DAl and histopathological scoring (Fig. 8). Our findings indicated that macrophage-derived p38a might be a promising target for colitis.

Accumulating data show that the activation of p38 MAPK signal is involved in intestinal inflammation and responsible for overactive immune system in IBD (Hommes, et al. 2002; ten Hove, et al. 2002; van Montfrans, et al. 2002). Inhibition of p38 MAPK can powerfully reduce the production of proinflammatory cytokines and reduce intestinal inflammation (Feng and Li 2011). Macrophages are considered as the gatekeepers of intestinal immune homeostasis and their dysregulation leads to the chronic inflammation in IBD (de Souza and Fiocchi 2016). In our study, we found that macrophagederived knockout of p38a improved experimental colitis by reducing the secretion of pro-inflammatory cytokines TNF- $a$, IL-1 $\beta$ and IL-6.

Intestinal barrier composed of mucous layer and epithelial cell layer can protect the intestinal mucosa from the attack of pathogens and antigens (Van der Sluis, et al. 2006). Previous study showed that muc-2 
deficiency in mice spontaneously induces the colitis (Van der Sluis, et al. 2006). In addition, IECs are interconnected by tight junctions (TJs), which regulate paracellular permeability. The members of claudin family are the principal constituents of the TJs, of which claudin-2 favors immune-mediated colitis and targeting claudin-2 relieved its progression. However, our results indicated that macrophage-derived knockout of p38a increased the enrichment of muc-2 and claudin-2 in experimental colitis, indicating that macrophage-derived knockout of p38a might improve experimental colitis by promoting mucosal homeostasis.

MAPKs are involved in multiple physiological processes, including cell growth, metabolism, differentiation and cell death (Huang, et al. 2009; Roux and Blenis 2004; Zhang and Liu 2002). Activation of p38 MAPK suppresses the proliferation of corneal endothelial cells, whereas the p38 MAPK inhibitor counteracts this effect (Nakahara, et al. 2018). P38a deficiency in neonatal muscle regulates cellular hyperproliferation and maturation (Perdiguero, et al. 2007). Neonatal mice lacking P38a in cardiac muscle exhibit an increase in DNA synthesis and mitosis in cardiomyocytes (Engel, et al. 2005). Herein, we investigated the effects of macrophage-derived p38a on colon cell proliferation and found that Ki-67 proliferation levels were markedly increased in experimental colitis in $\mathrm{p} 38 \mathrm{a}^{\Delta \mathrm{M} \varphi}$ group as compared with the WT group. In addition, increasing data indicate the pro-apoptotic role of p38 MAPKs in multiple cell types (De Zutter and Davis 2001; Ghatan, et al. 2000). We found that macrophage-derived knockout of p38a reduced cell apoptosis of IECs in DSS-induced colitis. Our results suggested that macrophagederived knockout of p38a might improve experimental colitis by regulating cell proliferation and apoptosis of IECs.

It has been widely accepted that the pathogenesis of IBD is associated with the imbalance of intestinal mucosal immune responses to environmental factors in genetically-predisposed individuals (Pizarro, et al.). Innate and adaptive immune responses cause inflammatory lesions in IBD (Geremia, et al. 2013). Of note, p38 MAPK acts a prominent role in innate and adaptive immune responses (Su, et al. 1994). Our results identified that immune process-related factors including Lgals9, Rtp4, Ddx60, Nlrp1b, Hsh2d, Oas2 and Oas3 were enriched in $\mathrm{p} 38 \mathrm{a}^{\Delta \mathrm{M} \varphi}$ group as compared with the WT group. Lgals9 is a $\beta$-galactosidebinding lectin that acts in physiological and pathological conditions, such as immune response (John and Mishra 2016). It induces T cell apoptosis (Kashio, et al. 2003; Zhu, et al. 2005) and dendritic cell (DC) activation (Li, et al. 2011). Lgals9 ${ }^{-/-}$mice harbors DSS-induced intestinal injury (Robinson, et al. 2020), (Kim, et al. 2010). RTP4 controls IFN-I response by affecting TBK1 and IRF3 phosphorylation (He, et al. 2020). DDX60 acts as a ligand-specific sentinel activated by RIG-1 to participate in innate immune response (Miyashita, et al. 2011; Oliver, et al. 2015). Nalp1b, a member of (NOD)-like receptors family recognizes pathogen-associated molecular patterns, such as lipopolysaccharide (Nour, et al. 2009). $\mathrm{HSH} 2 \mathrm{D}$ is a weighty signal molecule that involves in the activation of T cells ( $\mathrm{A}$, et al. 2001), leading to tumor malignancy and drug resistance in Tcell acute lymphoblastic leukaemia (Wang and Xiong 2020). The OASs are a family of IFN- and virus-induced proteins, consisting of OAS1, OAS2, OAS3, OASL (Liao, et al. 2020). OAS2 and OAS3 have antiviral activity (Li, et al. 2016; Liao, et al. 2020) and OAS3 negatively regulates chemokines and interferon-responsive factors in macrophages (Wook-Bin, et al. 2018). Our 
results further validated that Lgals9, Rtp4, Ddx60, Nlrp1b, Hsh2d, Oas2 and Oas3 were upregulated in p38a ${ }^{\Delta M \varphi}$ group as compared with the WT group, suggesting that macrophage-derived knockout of p38a might improve experimental colitis by regulating immune process.

Pharmacological inhibition of p38 MAPK has been used to treat various inflammatory diseases, such as rheumatoid arthritis, Alzheimer's disease and IBD (Waetzig, et al. 2002). SB203580 as a p38 MAPK inhibitor reduces the production of IFN- $y$ and IL-12p70 in colitis(ten Hove, et al. 2002). Herein, our results suggested that p38 inhibitors could significantly ameliorate experimental colitis. This may provide a potential theoretical basis for treatment of UC.

\section{Conclusion}

Taken together, we unveil a novel role of macrophage-derived p38a in colitis and found that macrophagederived p38a facilitates the pathogenesis of colitis by reducing inflammation response, protecting mucous barrier and regulating cell proliferation and immune process. Our work may provide a new approach for colitis treatment.

\section{Declarations}

\section{Ethics approval and consent to participate}

All experiments involving animals were conducted according to the ethical policies and procedures approved by the ethics committee of Shanghai Sixth People's Hospital (2020-0015). All subjects provided written informed consent and the study protocol was approved by the ethics committee of Shanghai Sixth People's Hospital. (2020-231).

\section{Consent for publication}

All the authors agreed to publish articles in the journal.

\section{Availability of data and material}

The data underlying this article will be shared on reasonable request to the corresponding author.

\section{Funding}

Jinshui Zhu received grants from National Natural Science Foundation of China (No.82074161), Clinical research project of Shanghai Health Committee (ZHYY-ZXYJHZX-202013), and Western medicine guidance project of Shanghai Science and Technology Commission (17411966500).

Jing Zhang received grants from Double-Hundred Talent Plan of Shanghai Jiao Tong University School of Medicine (No. 20191831) and National Natural Science Foundation of China (No. 81873143). 
Not applicable.

\section{Authors' contributions}

JZ and JSZ supervised and designed the study and WC drafted the manuscript. WC, HF and RL performed the experiments. WC, XC and YY conducted the statistical analysis. WC wrote the paper and JZ revised the paper. All authors read and approved the final manuscript.

\section{Competing interests}

All authors declare that they have no conflicts of interest.

\section{References}

1. A, Tsukasa Oda, et al. 2001 HSH2: A Novel SH2 Domain-Containing Adapter Protein Involved in Tyrosine Kinase Signaling in Hematopoietic Cells. Biochemical and Biophysical Research Communications 288(5):1078-1086.

2. Cha, H, et al. 2017 Increased susceptibility of IDH2-deficient mice to dextran sodium sulfate-induced colitis. Redox biology 13:32-38.

3. Chang, CJ, et al. 2019 Change in Renal Glomerular Collagens and Glomerular Filtration BarrierRelated Proteins in a Dextran Sulfate Sodium-Induced Colitis Mouse Model. International journal of molecular sciences 20(6).

4. Chen, GY, et al. 2011 A functional role for Nlrp6 in intestinal inflammation and tumorigenesis. Journal of immunology (Baltimore, Md. : 1950) 186(12):7187-94.

5. Chen, W, et al. 2019 Taraxacum officinale extract ameliorates dextran sodium sulphate-induced colitis by regulating fatty acid degradation and microbial dysbiosis. Journal of cellular and molecular medicine 23(12):8161-8172.

6. de Souza, HS, and C Fiocchi 2016 Immunopathogenesis of IBD: current state of the art. Nature reviews. Gastroenterology \& hepatology 13(1):13-27.

7. De Zutter, GS, and RJ Davis 2001 Pro-apoptotic gene expression mediated by the p38 mitogenactivated protein kinase signal transduction pathway. Proceedings of the National Academy of Sciences of the United States of America 98(11):6168-73.

8. Engel, FB, et al. 2005 p38 MAP kinase inhibition enables proliferation of adult mammalian cardiomyocytes. Genes \& development 19(10):1175-87.

9. Feng, YJ, and YY Li 2011 The role of p38 mitogen-activated protein kinase in the pathogenesis of inflammatory bowel disease. Journal of digestive diseases 12(5):327-32.

10. Geremia, Alessandra, et al. 2013 Innate and adaptive immunity in inflammatory bowel disease. Autoimmunity reviews 13(1).

11. Ghatan, S, et al. 2000 p38 MAP kinase mediates bax translocation in nitric oxide-induced apoptosis in neurons. The Journal of cell biology 150(2):335-47. 
12. Gupta, J, et al. 2014 Dual function of p38a MAPK in colon cancer: suppression of colitis-associated tumor initiation but requirement for cancer cell survival. Cancer cell 25(4):484-500.

13. Hanauer, Stephen B Inflammatory bowel disease: epidemiology, pathogenesis, and therapeutic opportunities. Inflammatory Bowel Diseases (suppl_1):S3-S9.

14. He, Xiao, et al. 2020 RTP4 inhibits IFN-I response and enhances experimental cerebral malaria and neuropathology. Proceedings of the National Academy of ences 117(32):202006492.

15. Hommes, D, et al. 2002 Inhibition of stress-activated MAP kinases induces clinical improvement in moderate to severe Crohn's disease. Gastroenterology 122(1):7-14.

16. Huang, Gonghua, Lewis Zhichang Shi, and Hongbo Chi 2009 Regulation of JNK and p38 MAPK in the immune system: Signal integration, propagation and termination. Cytokine 48(3):161-169.

17. Hui, L, et al. 2007 p38alpha suppresses normal and cancer cell proliferation by antagonizing the JNK-c-Jun pathway. Nature genetics 39(6):741-9.

18. Jiang, $Y$, et al. 1997 Characterization of the structure and function of the fourth member of p38 group mitogen-activated protein kinases, p38delta. The Journal of biological chemistry 272(48):30122-8.

19. Jing, Y, et al. 2015 Hepatic p38a regulates gluconeogenesis by suppressing AMPK. Journal of hepatology 62(6):1319-27.

20. John, Sebastian, and Rashmi Mishra 2016 Galectin-9: From cell biology to complex disease dynamics. J Bio 41(3):507-534.

21. Kashio, Y., et al. 2003 Galectin-9 Induces Apoptosis Through the Calcium-Calpain-Caspase-1 Pathway. Journal of Immunology 170(7):3631-3636.

22. Kim, C, et al. 2008 The kinase p38 alpha serves cell type-specific inflammatory functions in skin injury and coordinates pro- and anti-inflammatory gene expression. Nature immunology 9(9):101927.

23. Kim, Joo Young, et al. 2010 Inhibition of dextran sulfate sodium (DSS)-induced intestinal inflammation via enhanced IL-10 and TGF- $\beta$ production by galectin- 9 homologues isolated from intestinal parasites. Mol Biochem Parasitol 174(1):53-61.

24. Kumar, S, J Boehm, and JC Lee 2003 p38 MAP kinases: key signalling molecules as therapeutic targets for inflammatory diseases. Nature reviews. Drug discovery 2(9):717-26.

25. Li, Yize, et al. 2016 Activation of RNase $L$ is dependent on OAS3 expression during infection with diverse human viruses. Proc Natl Acad U S A 113(8):2241-2246.

26. Li, Yurong, et al. 2011 The $\mathrm{N}$ - and C-terminal carbohydrate recognition domains of galectin-9 contribute differently to its multiple functions in innate immunity and adaptive immunity. Molecular Immunology 48(4):670-677.

27. Liao, X, et al. 2020 2', 5'-Oligoadenylate Synthetase 2 (OAS2) Inhibits Zika Virus Replication through Activation of Type I IFN Signaling Pathway. Viruses 12(4).

28. Liu, J, et al. 2017 Deficiency of p38a in macrophage ameliorates d-galactosamine/TNF-a-induced acute liver injury in mice. The FEBS journal 284(24):4200-4215. 
29. Michielan, A, and R D'Incà 2015 Intestinal Permeability in Inflammatory Bowel Disease: Pathogenesis, Clinical Evaluation, and Therapy of Leaky Gut. Mediators of inflammation 2015:628157.

30. Miyashita, M., et al. 2011 DDX60, a DEXD/H Box Helicase, Is a Novel Antiviral Factor Promoting RIG-ILike Receptor-Mediated Signaling. Molecular and Cellular Biology 31(18):3802-3819.

31. Nakahara, M, et al. 2018 Effect of a p38 Mitogen-Activated Protein Kinase Inhibitor on Corneal Endothelial Cell Proliferation. Investigative ophthalmology \& visual science 59(10):4218-4227.

32. Nour, Adel M., et al. 2009 Anthrax lethal toxin triggers the formation of a membrane-associated inflammasome complex in murine macrophages. Infection \& Immunity 77(3):1262-1271.

33. Oliver, et al. 2015 DDX60L Is an Interferon-Stimulated Gene Product Restricting Hepatitis C Virus Replication in Cell Culture. Journal of Virology.

34. Otsuka, $M$, et al. 2010 Distinct effects of p38alpha deletion in myeloid lineage and gut epithelia in mouse models of inflammatory bowel disease. Gastroenterology 138(4):1255-1265.

35. Perdiguero, E, et al. 2007 Genetic analysis of p38 MAP kinases in myogenesis: fundamental role of p38alpha in abrogating myoblast proliferation. The EMBO journal 26(5):1245-56.

36. Pizarro, Theresa T, et al. Challenges in IBD Research: Preclinical Human IBD Mechanisms. Inflammatory Bowel Diseases (Supplement_2):Supplement_2.

37. Robinson, Brian S., et al. 2020 Galectin-9 Is a Novel Regulator of Epithelial Restitution. American Journal Of Pathology 190(8).

38. Roux, P. P., and J. Blenis 2004 ERK and p38 MAPK-Activated Protein Kinases: a Family of Protein Kinases with Diverse Biological Functions. Microbiology and Molecular Biology Reviews 68(2):320344.

39. Shi, Y, et al. 2018 Smad nuclear interacting protein 1 (SNIP1) inhibits intestinal inflammation through regulation of epithelial barrier function. Mucosal immunology 11(3):835-845.

40. Souza, Heitor S P De, and Claudio Fiocchi 2016 Immunopathogenesis of IBD: current state of the art. Nature Reviews Gastroenterology \& Hepatology 13(1):13-27.

41. Su, Bing, et al. 1994 JNK is involved in signal integration during costimulation of T lymphocytes. Cell 77(5):727-736.

42. ten Hove, T, et al. 2002 Dichotomal role of inhibition of p38 MAPK with SB 203580 in experimental colitis. Gut 50(4):507-12.

43. Van der Sluis, M, et al. 2006 Muc2-deficient mice spontaneously develop colitis, indicating that MUC2 is critical for colonic protection. Gastroenterology 131(1):117-29.

44. van Montfrans, C, et al. 2002 Inflammatory signal transduction in Crohn's disease and novel therapeutic approaches. Biochemical pharmacology 64:789-95.

45. Waetzig, G. H., et al. 2002 p38 mitogen-activated protein kinase is activated and linked to TNF-alpha signaling in inflammatory bowel disease. Journal of Immunology 168(10):5342-5351. 
46. Wang, Hongjian, et al. 2018 CircSLC3A2 functions as an oncogenic factor in hepatocellular carcinoma by sponging miR-490-3p and regulating PPM1F expression. Molecular Cancer 17(1).

47. Wang, J, and $Y$ Xiong $2020 \mathrm{HSH} 2 \mathrm{D}$ contributes to methotrexate resistance in human T-cell acute lymphoblastic leukaemia. Oncology reports 44(5):2121-2129.

48. Wang, L, et al. 2017 rSj16 Protects against DSS-Induced Colitis by Inhibiting the PPAR-a Signaling Pathway. Theranostics 7(14):3446-3460.

49. Weigmann, et al. 2017 Chemically induced mouse models of acute and chronic intestinal inflammation. Nature Protocols Erecipes for Researchers.

50. Wook-Bin, et al. 2018 OAS1 and OAS3 negatively regulate the expression of chemokines and interferon-responsive genes in human macrophages. Bmb Reports.

51. Xavier, RJ, and DK Podolsky 2007 Unravelling the pathogenesis of inflammatory bowel disease. Nature 448(7152):427-34.

52. Zhang, W, and HT Liu 2002 MAPK signal pathways in the regulation of cell proliferation in mammalian cells. Cell research 12(1):9-18.

53. Zhang, $X$, et al. 2019 Macrophage p38a promotes nutritional steatohepatitis through M1 polarization. Journal of hepatology 71(1):163-174.

54. Zhu, C, et al. 2005 The Tim-3 ligand galectin-9 negatively regulates T helper type 1 immunity. Nature immunology 6(12):1245-52.

\section{Tables}

\section{Table 1. List of primers of the genes.}




\begin{tabular}{lll} 
Gene & Forward primer & Reverse primer \\
\hline GAPDH & CCTCGTCCCGTAGACAAAATG & TGAGGTCAATGAAGGGGTCGT \\
\hline Lgals9 & CTCTTCAGTGCCCAGTCTCCA & CTGTCCGTTCTGCTTCGTGT \\
\hline Rtp4 & GAATGCTGTTCCCCGATGAC & GGAACACTGGAACCTGCCAAG \\
\hline Ddx60 & CTACTAGCTCCTCATCGCCAA & CTCCCAGATTTCCGCTCCA \\
\hline Nlrp1b & AATCCTATGAGGCAGTGCGG & GCCAGGCTCCTATCAATGTCA \\
\hline Hsh2d & ACACCCTTTCCTACAAAGCCC & CTCCCCGAATGGCCGAATAG \\
\hline Oas2 & GAAGAAGCGAAGGAGTGGCT & GGGGGTCTGCATTACCTAGAC \\
\hline Oas3 & CCTGAGTTCACAGCAGCCGTA & CCCTTGGCAATCCTTATCACT \\
\hline mucin-1 & AAGTTCAGGTCAGGCTCCGT & GGGAGGGAACTGCATCTCATT \\
\hline mucin-2 & AGAAGCCAGATCCCGAAACCA & TCTCGGCAGTCAGACGCAAA \\
\hline Z0-1 & CAAAGCCCACCAAGGTCACAC & TTCAGTAAGGTAGCCCGCTCA \\
\hline occludin & TAAAAGGGCTCCCACGAAGG & GGATATTCCCTGACCCAGTCC \\
\hline claudin-1 & ATTTCAGGTCTGGCGACATTAG & ACAGGAGCAGGAAAGTAGGACA \\
\hline claudin-2 & AGTGCGATATCTACAGTACCCTT & ATAATACAAGCCAGCGAGGACA
\end{tabular}

\section{Figures}




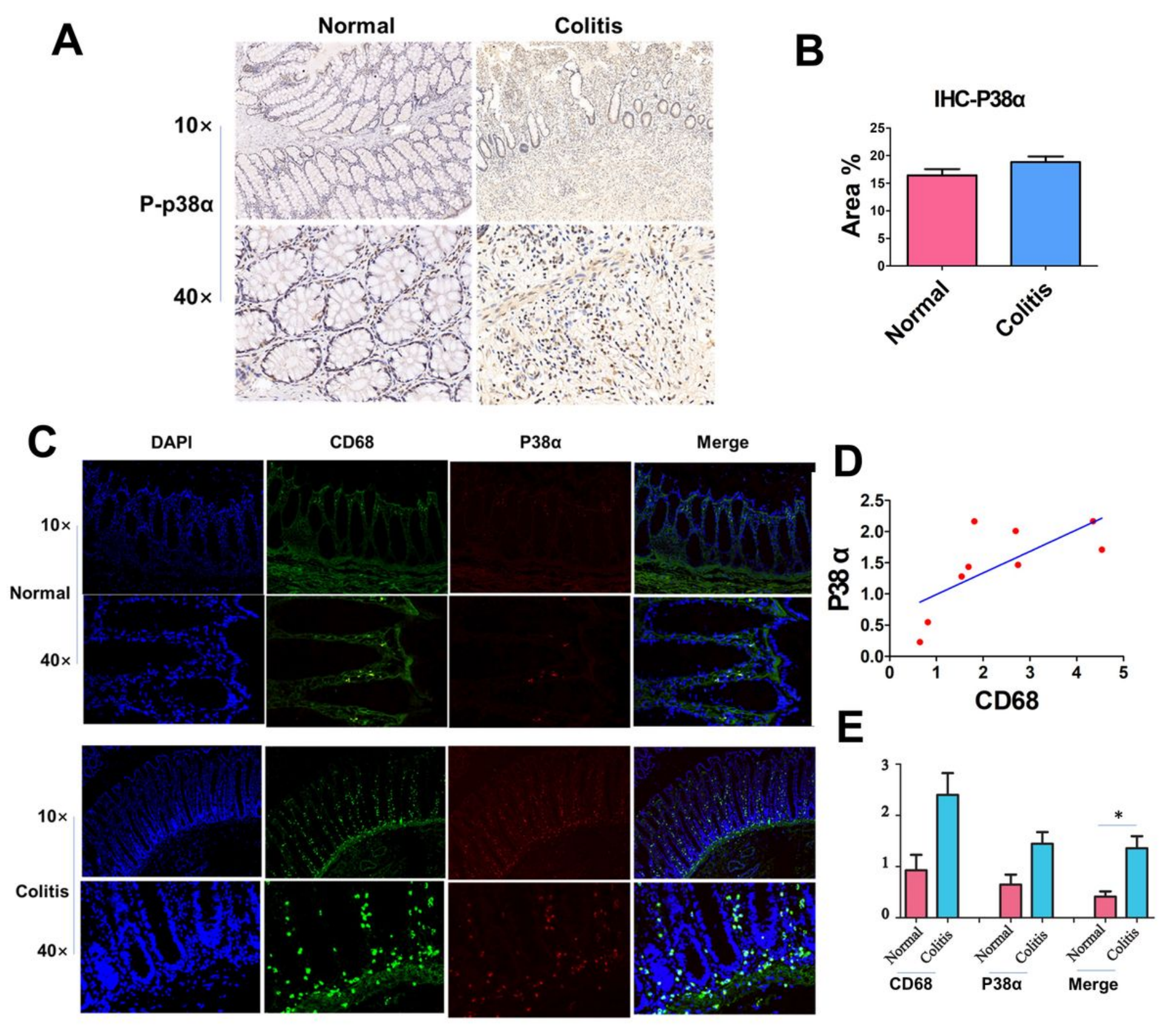

\section{Figure 1}

Macrophage p38a is upregulated in human colitis colon tissues. (A) Representative immunohistochemistry images of p-p38a in colitis and normal human colon tissues. (B) p-p38a protein expression in colitis $(n=10)$ and normal human liver tissues $(n=5)$. (C) Representative FISH images of CD68 and p38a in colitis and normal human colon tissues. (D) Analysis of the association between CD68 and p38a in human colitis colon tissues. (E) The expression of CD68, p38a and CD68-p38a in colitis $(n=10)$ and normal human liver tissues $(n=5) . * P<0.05$ 
A

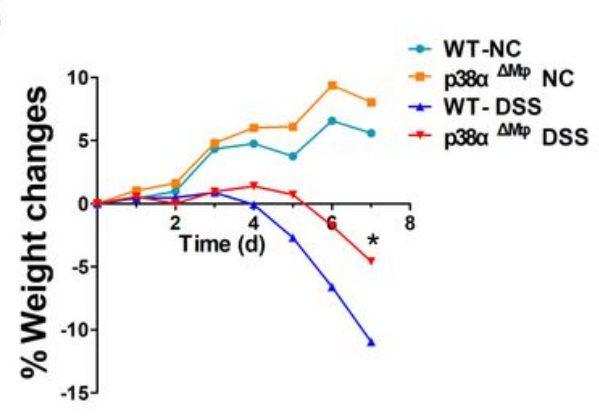

D

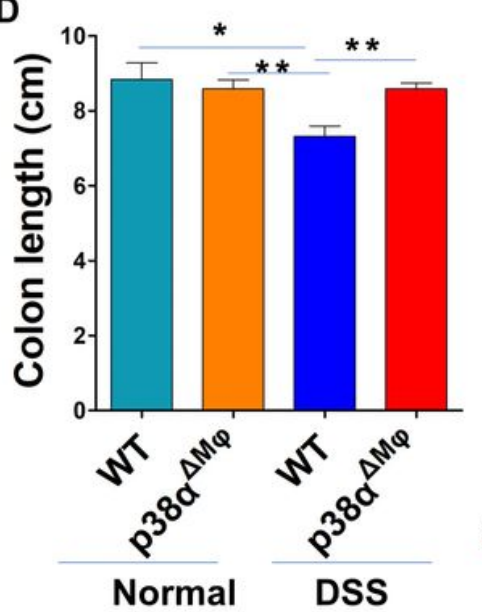

B

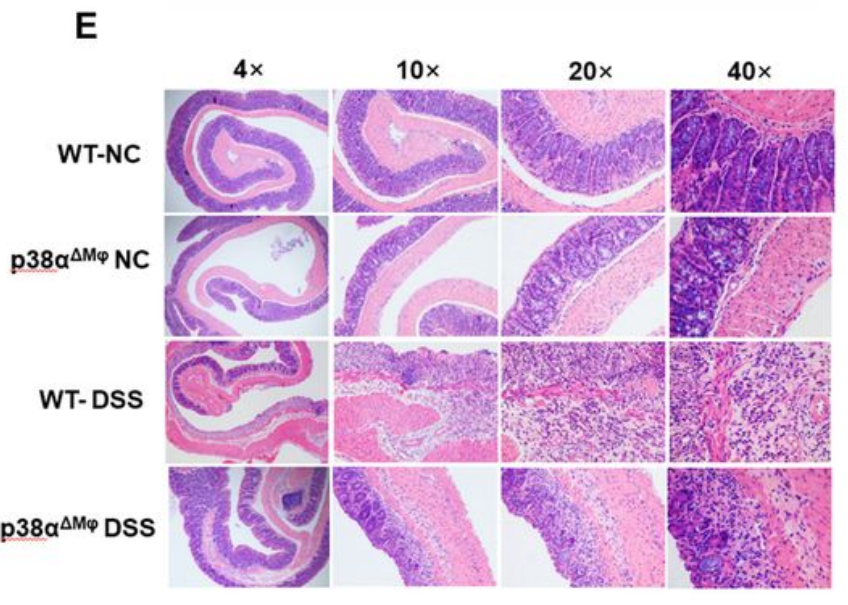

C
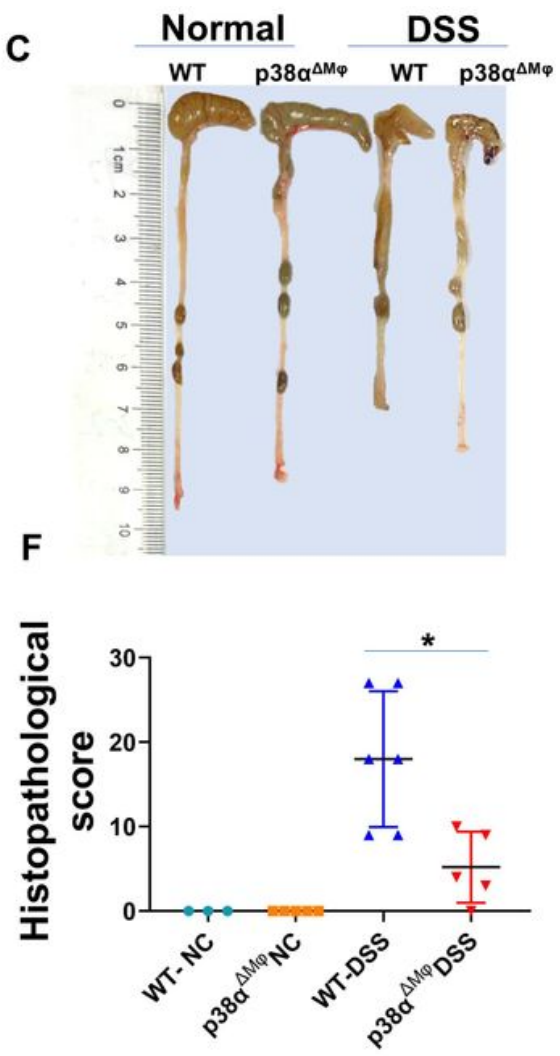

Figure 2

Macrophage p38a protects against DSS-induced colitis. (A) Body weight changes of WT and p38a $\Delta M$ Mmice were monitored daily. (B) DAl of WT and p38a $\Delta M \varphi$ mice were monitored daily. (C) Gross morphology images of colons of WT and p38a $\Delta M \varphi$ mice. (D) Colon lengths of WT and p38a $\Delta M \varphi m i c e$.

(E) Representative $H \& E$ staining of distal colon sections of WT and $\mathrm{p} 38 \mathrm{a} \Delta \mathrm{M} \varphi$ mice. (F) Histology scores of WT and $\mathrm{p} 38 \mathrm{a} \Delta \mathrm{M}$ pmice. Data are showed as means \pm SEM. $* \mathrm{P}<0.05$ and ${ }^{* * P}<0.01$. 
A
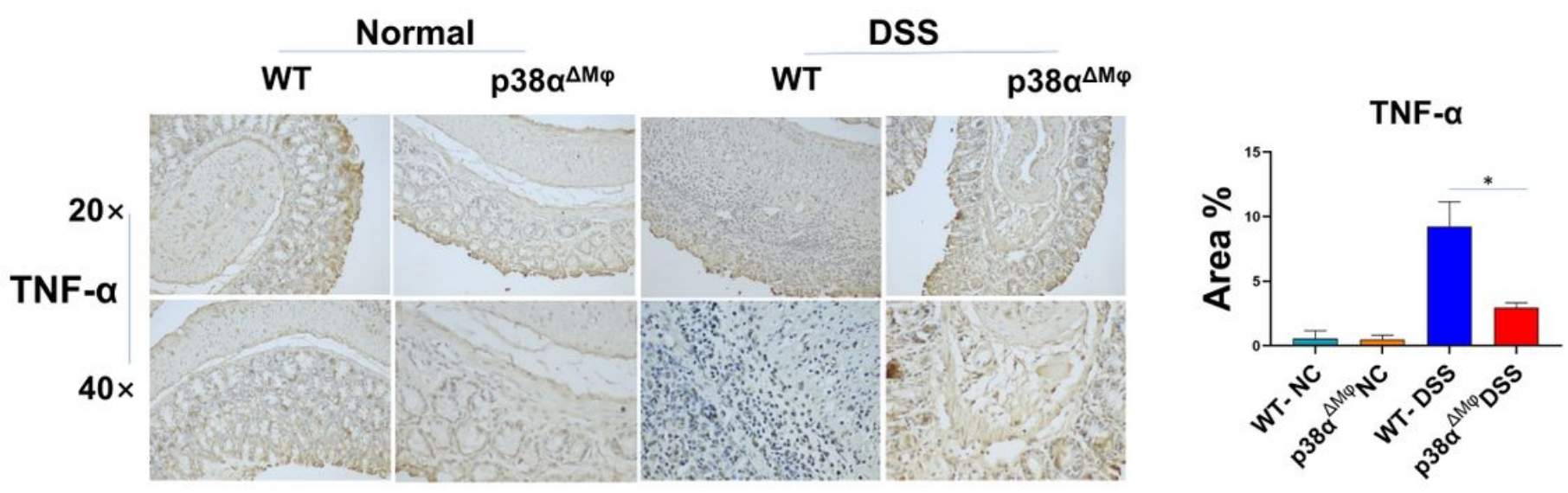

B

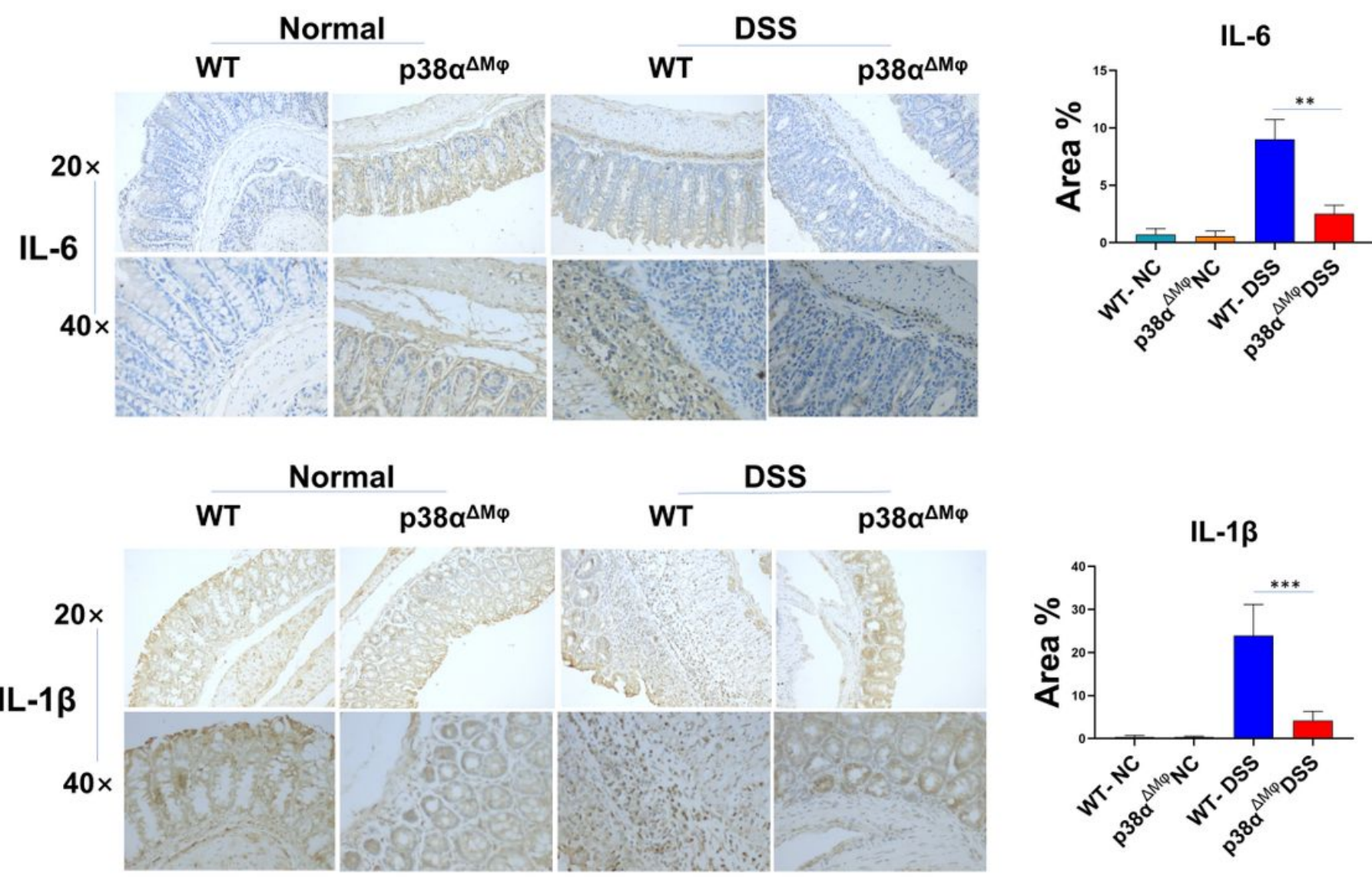

Figure 3

Macrophage p38a deletion inhibits pro-inflammatory cytokine production in DSS induced colitis. (A-C) The expression levels of TNF - $a, I L-6$ and IL- $1 \beta$ in colon tissues were analyzed by IHC analysis. ${ }^{*}<<0.05$, $\star \star P<0.01, * \star * P<0.001$. 


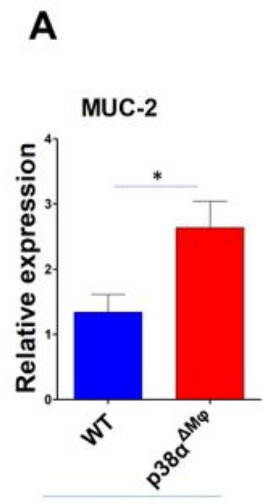

DSS

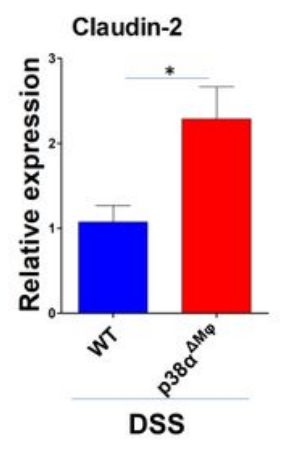

Claudin-2

$40 \times$
B

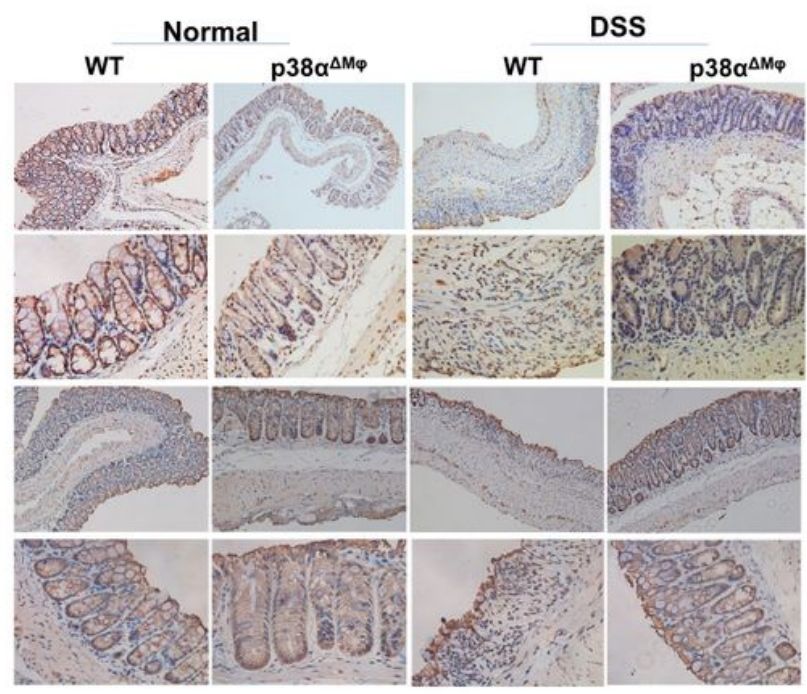

D

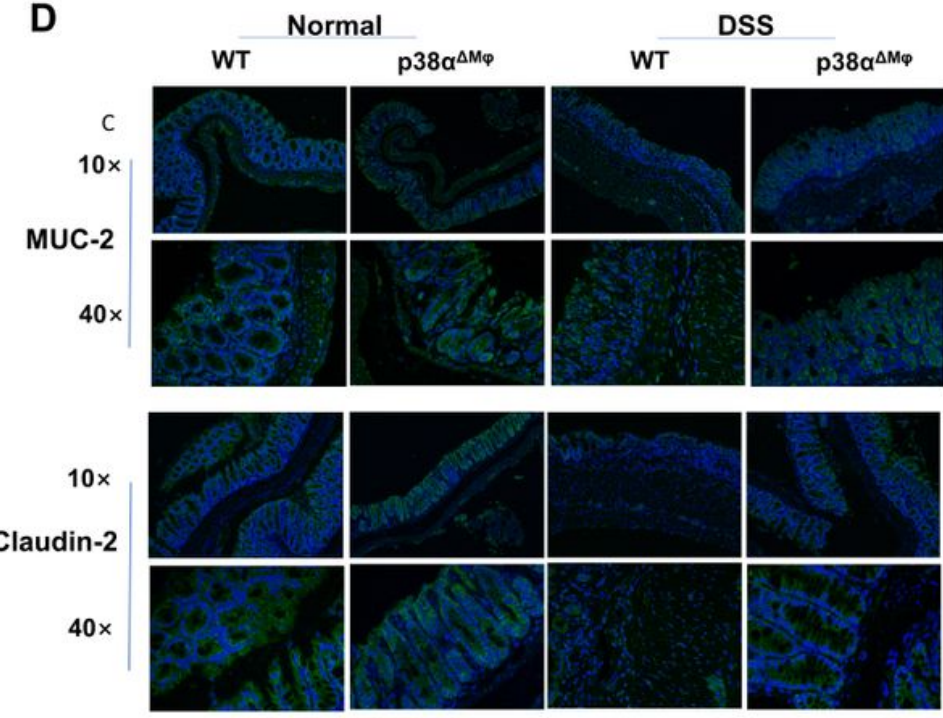

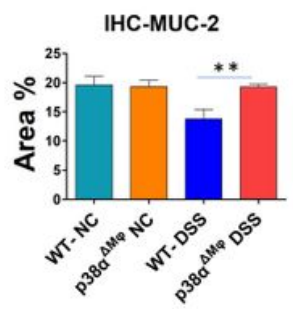

IHC-Claudin-2
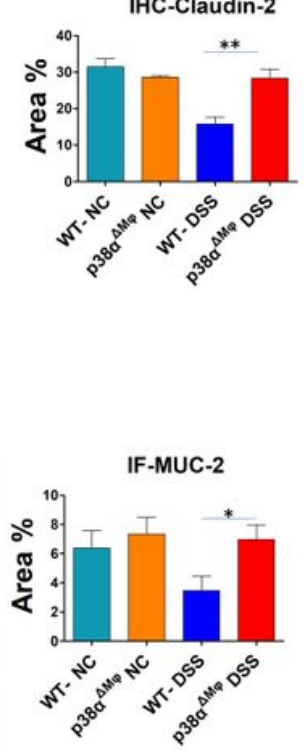

IF-Claudin-2

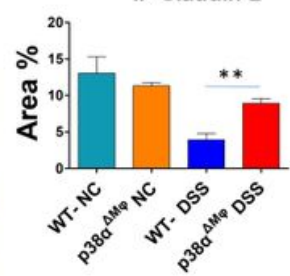

\section{Figure 4}

Macrophage p38a deficiency induces the secretion of muc-2 and claudin-2 (A) The relative mRNA expression of muc-2 and claudin-2 in colon tissues of DSS-treated WT and p38a $\triangle \mathrm{M} \varphi$ mice were analyzed by qRT-PCR. (B) Representative IHC images of muc-2 and claudin-2 in WT and p38a $\triangle M \varphi$ mice. (C) Western blot of claudin-2 protein levels in colonic tissues of DSS-treated WT and p38a $\Delta \mathrm{M} \varphi$ mice. (D) Representative IF images of muc-2 and claudin-2 in WT and $\mathrm{p} 38 \mathrm{a} \Delta \mathrm{M} \varphi$ mice. Data are showed as means \pm SEM. * $P<0.05$ and ** $P<0.01$. 
A

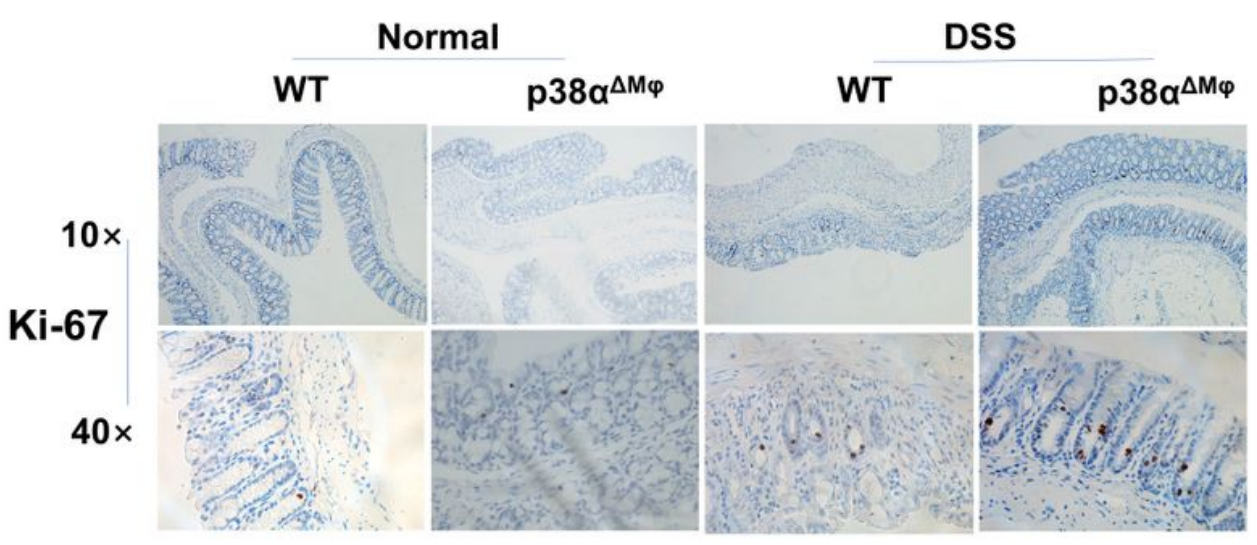

B
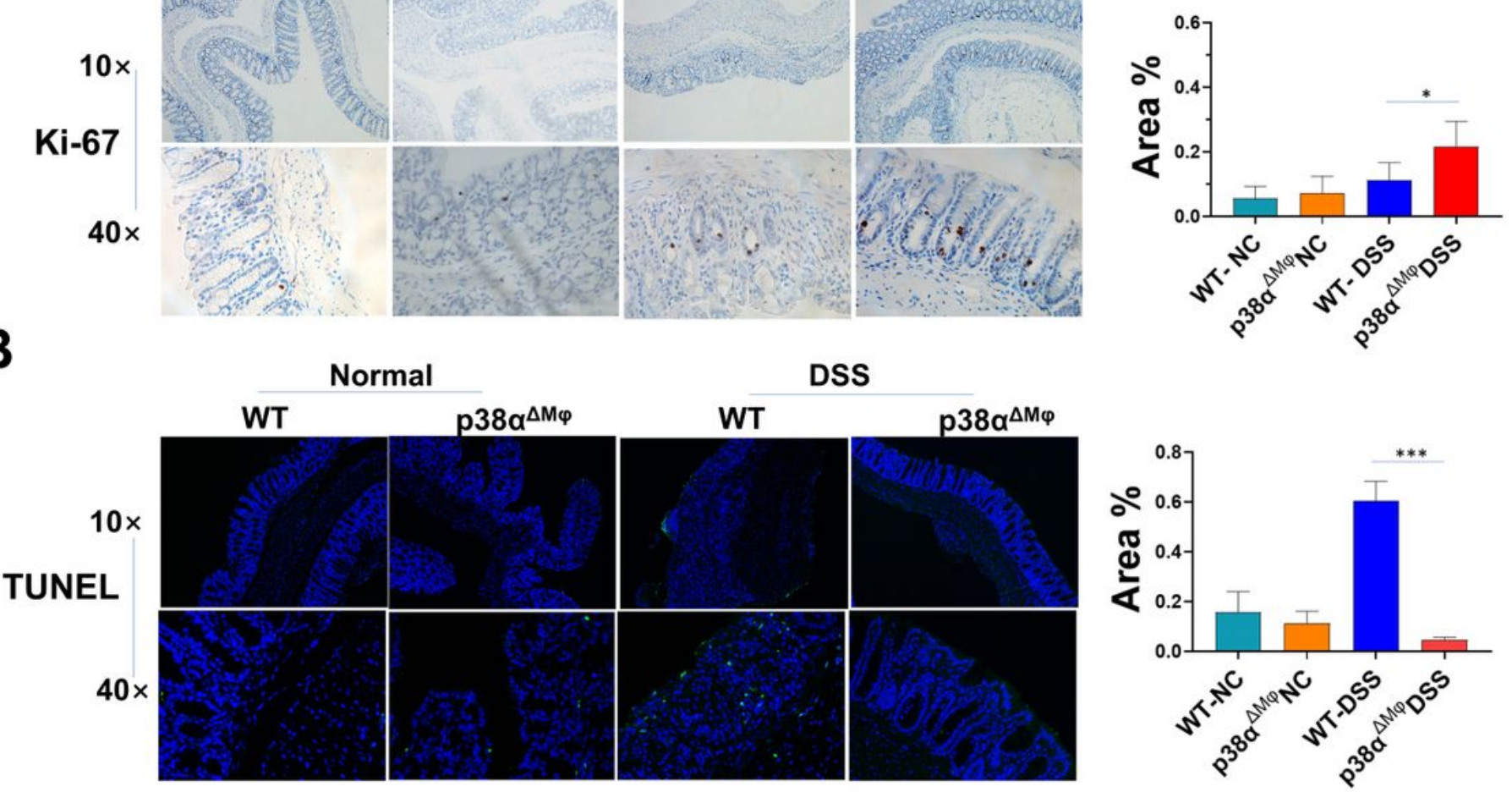

Figure 5

Macrophage p38a deficiency affects the proliferation and apoptosis of IECs and thus inhibits the course of colitis. (A) Ki-67 expression in WT and p38a $\Delta \mathrm{M} \varphi$ mice was detected by immunohistochemistry. (B) The apoptosis of IECs was detected by TUNEL. Data are showed as means \pm SEM. $* P<0.05$ and $* * * P<$ 0.001 . 
A

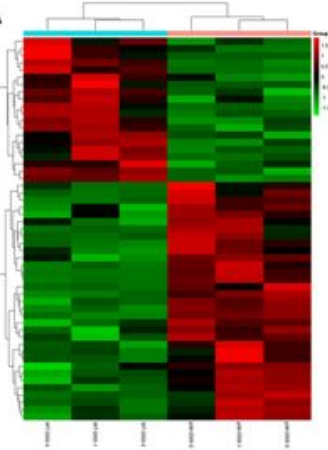

D

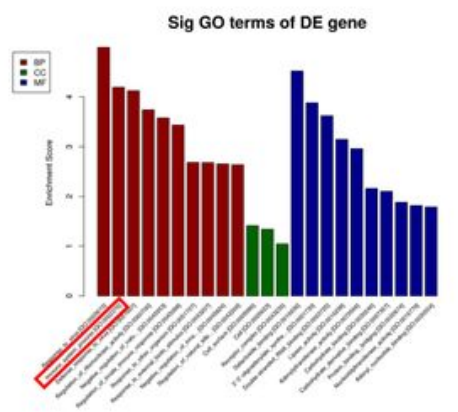

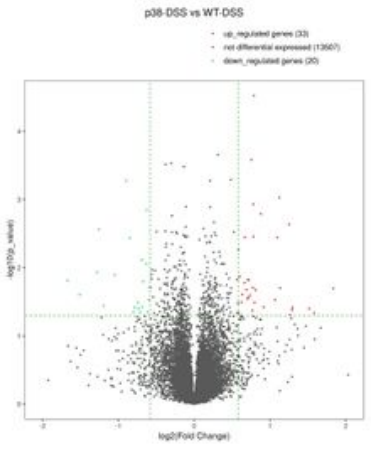

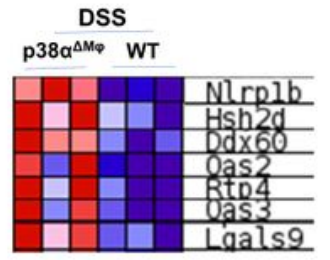

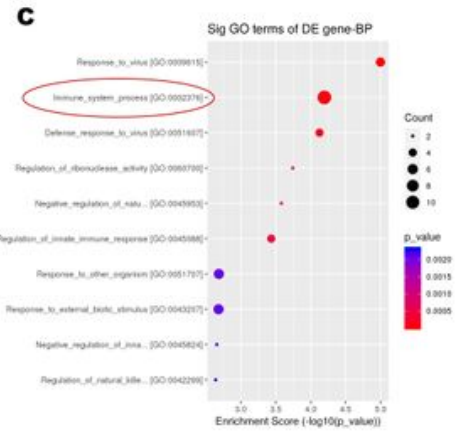

F

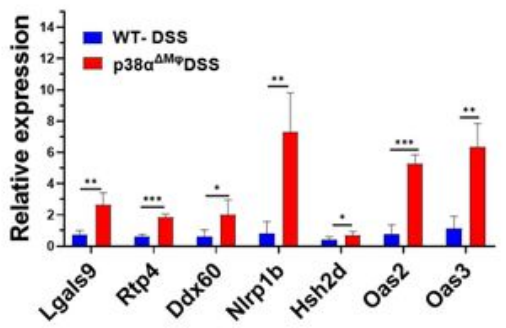

G
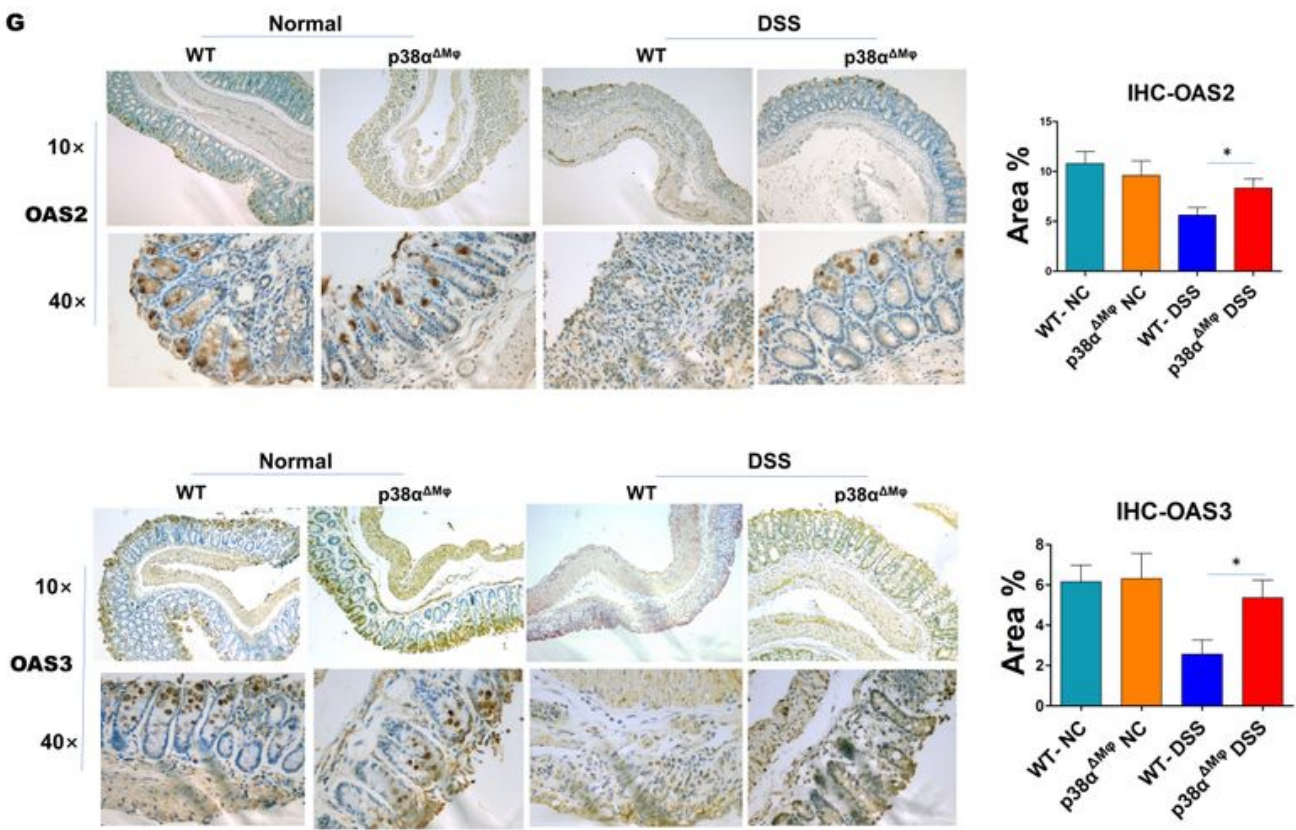

\section{Figure 6}

Macrophage p38a deficiency enhances immune system process during colitis (A) Heatmap of the differentially expressed genes between WT and $\mathrm{p} 38 \mathrm{a} \triangle \mathrm{M} \varphi$ mice groups after DSS treatment. (B) Volcano plotting of the differentially expressed genes between WT and p38a $\triangle M \varphi$ mice groups after DSS treatment. (C凶D) Go analysis was performed for genes with significant difference in expression. (E) The major genes that related to immune system process. (F) The mRNA expression of Lgals9, Rtp4, Ddx60, 
Nlrp1b, Hsh2d, Oas2 and Oas3 in colonic tissues of WT and p38a $\Delta \mathrm{M} \varphi$ mice on day 7 after DSS treatment were determined by real-time PCR. (G) The protein expression of Oas2 and Oas3 in colonic tissues of WT and p38a $\triangle M \varphi$ mice were determined by immunohistochemistry. Data are showed as means \pm SEM. ${ }^{*} \mathrm{P}<0.05, * * \mathrm{P}<0.01$, and $* * * \mathrm{P}<0.001$.
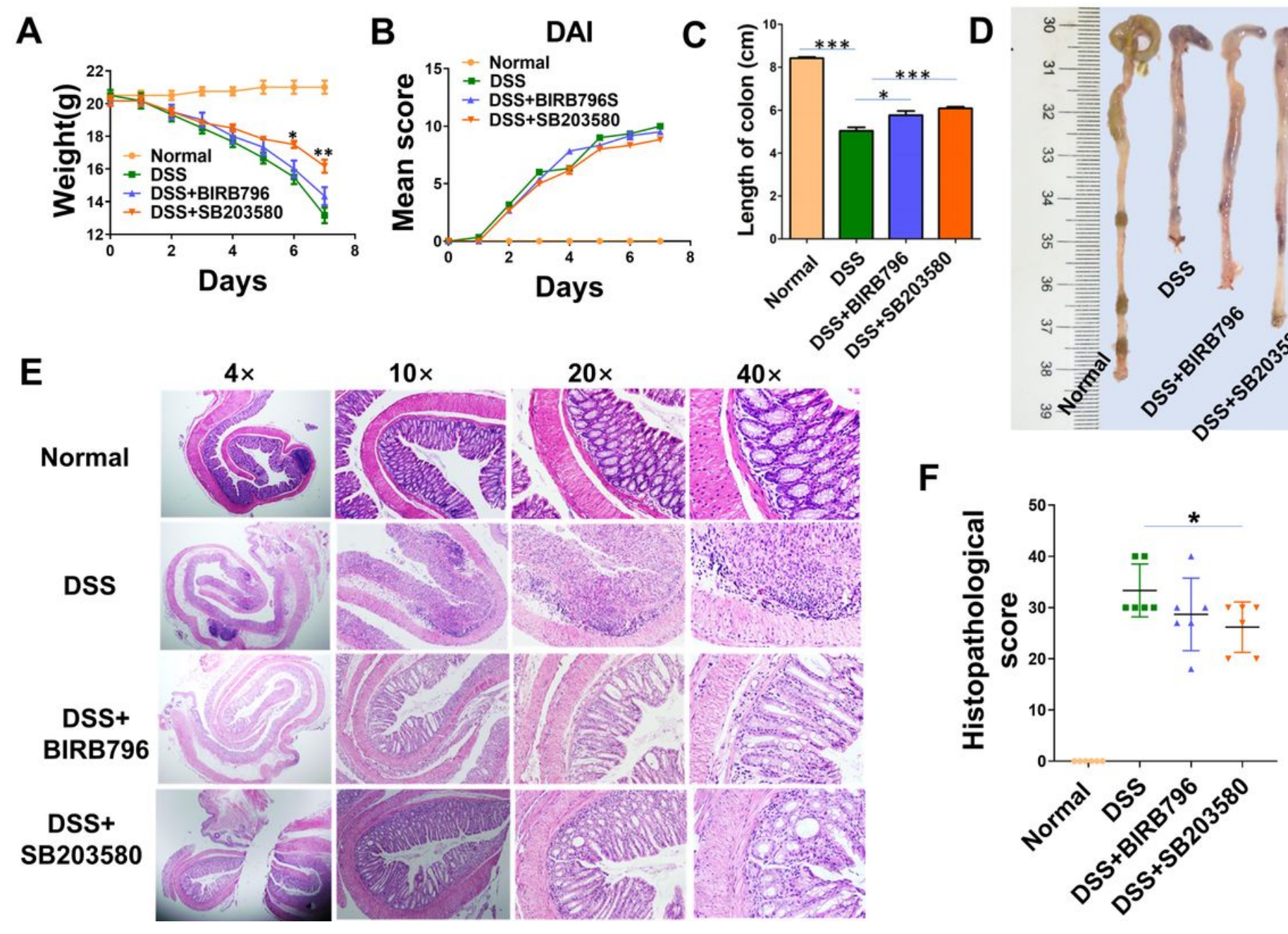

\section{Figure 7}

The administration of p38 MAPK inhibitor restricts colitis in mice (A) Body weights of the four groups were monitored daily. (B) DAl scores of the four groups were recorded in each mice. (C) Colon lengths of each mice were calculated. (D) Representative pictures of colons from the four groups. (E) Representative H\&E staining of distal colon sections of the four groups. (F) Histological scores of colon tissues were calculated. Data are showed as means \pm SEM. $* P<0.05$, $* * P<0.01$, and $* \star * P<0.001$. 


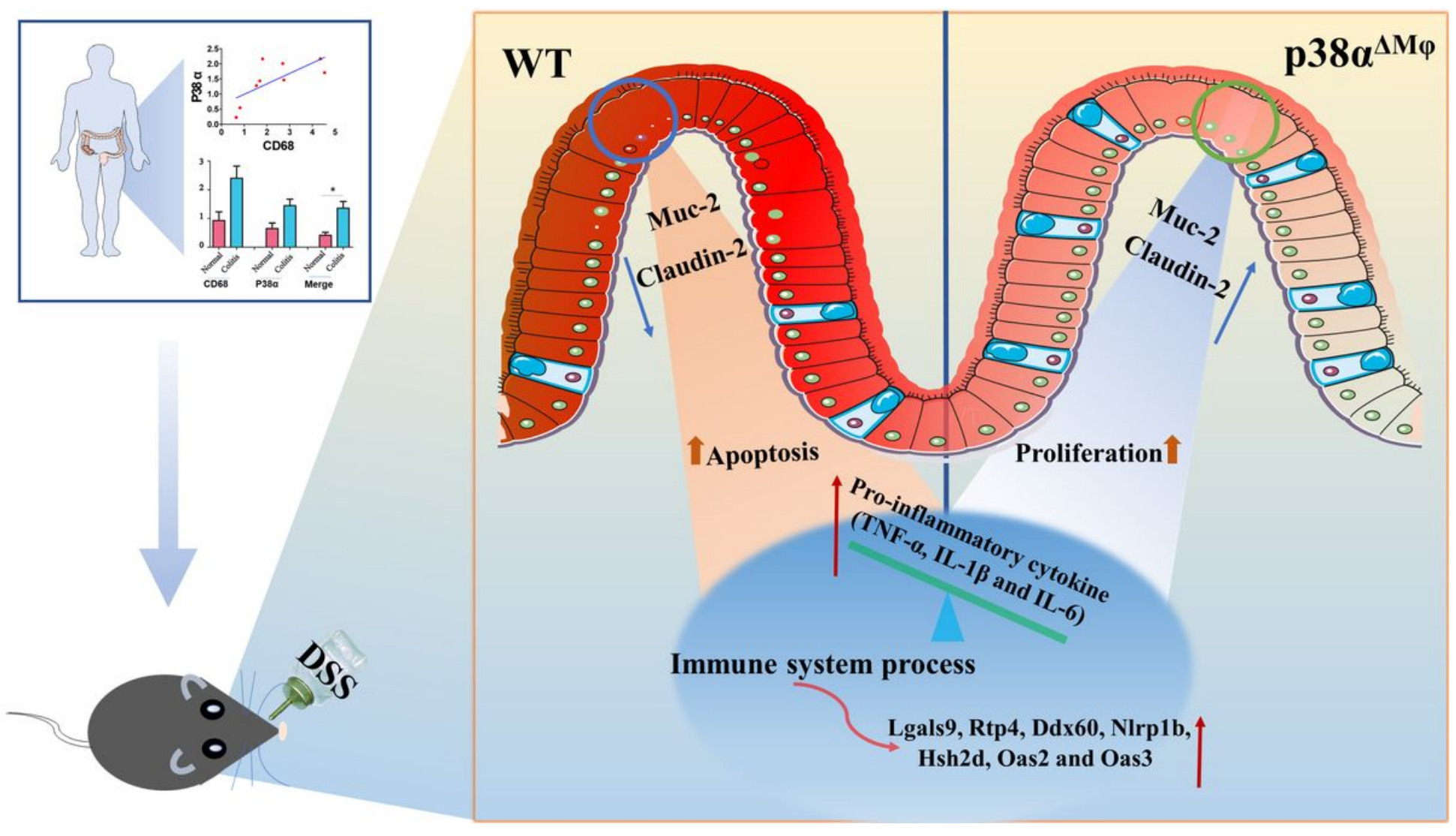

\section{Figure 8}

It has been reported that macrophage-derived p38a promotes liver injury and steatohepatitis progression. Herein, we estimated the role of macrophage-derived p38a in DSS-induced experimental colitis. We found that the expression of macrophage-derived p38a was elevated in colitis tissues, and macrophage-specific knockout of p38a improved the clinical symptoms, DAl and histopathological scoring

\section{Supplementary Files}

This is a list of supplementary files associated with this preprint. Click to download.

- Supplementaryfile.docx 